\title{
Testing quantitative models of backward masking
}

\author{
GREGORY FRANCIS \\ Purdue University, West Lafayette, Indiana \\ and \\ MICHAEL H. HERZOG \\ University of Bremen, Bremen, Germany
}

\begin{abstract}
We analyzed the relationship between U-shaped and monotonic-shaped masking functions, using both computer simulations of quantitative models and experimental data. Our analysis revealed that quantitative models of backward masking predict that U-shaped masking functions should appear for weak masks and monotonic masking functions should appear for strong masks. The models predict, moreover, that for a fixed target and experimental task, as the mask changes it is possible to go from U-shaped to monotonic-shaped masking functions. Significantly, the models predict that at each stimulus onset asynchrony between the target and the mask, the U-shaped function must have weaker masking than the monotonic-shaped function. Contrary to the predictions of the models, we show an experimental situation that generates masking functions that violate this prediction.
\end{abstract}

Backward masking refers to impaired performance on some judgment of a target stimulus when it is followed by a mask. In visual backward masking, both the target and the mask stimuli are usually very brief (often less than $50 \mathrm{msec}$ ). The target stimulus and the task are always set so that if the target stimulus is presented by itself, it is easy for observers to perform whatever judgment about the target is required. However, presentation of a mask stimulus, even $100 \mathrm{msec}$ after the target has turned off, can make the observer's task exceedingly difficult. In some cases, observers report not seeing the target at all. Backward masking is a fundamental tool in cognitive psychology and vision research, where it is used to limit the amount of information processing (see recent reviews by Breitmeyer \& Ogmen, 2000, and Enns \& Di Lollo, 2000). Backward masking is also used to investigate aspects of schizophrenia (e.g., Braff \& Saccuzzo, 1981; Green, Nuechterlein, \& Mintz, 1994; Slaghuis \& Bakker, 1995).

Often, the properties of the target and mask stimuli are held fixed, but the stimulus onset asynchrony (SOA), the time between target onset and mask onset, is varied. The resulting set of data is called a masking function. One particularly interesting characteristic of backward masking is that the masking function is sometimes U-shaped. For short SOAs, the target is clearly seen, and the required

G. F. was supported by a fellowship at the Hanse Wissenschaftskolleg, Delmenhorst, Germany. M.H.H. was supported by the SFB 517 "Neurocognition" of the Deutsche Forschungsgemeinschaft (DFG). This material is based on work supported by National Science Foundation Grant 0108905. Correspondence concerning this article should be addressed to G. Francis, Department of Psychological Sciences, Purdue University, 703 Third Street, West Lafayette, IN 47907-2004 (e-mail: gfrancis@psych.purdue.edu). task is fairly easy to perform. For middle duration SOAs (around $80 \mathrm{msec}$, but it varies substantially), the target is harder to see, and the task is difficult to perform. For long SOAs, the task performance is again quite good, perhaps because the target is partially processed before the mask appears. U-shaped masking functions are not always observed, however. In some cases the strongest masking occurs when the target and the mask are presented at the same time $(\mathrm{SOA}=0)$, and masking effects grow weaker as the SOA between the target and the mask increases. Under such situations, a monotonic-shaped masking function is found.

The differences between monotonic- and U-shaped masking functions have generated much discussion about theories and interpretations of masking. Kolers (1962) noted that monotonic masking functions appeared for high-energy masks and U-shaped masking functions appeared for masks that were similar in energy to the target. Subsequent work showed that this observation holds for a variety of mask types, including pattern masks that have contours overlapping the target (e.g., Hellige, Walsh, Lawrence, \& Prasse, 1979; Spencer \& Shuntich, 1970; Turvey, 1973), metacontrast masks that do not overlap the target (e.g., Schiller, 1965; Weisstein, 1972), and masks consisting of a homogenous field or disk that covers the target (e.g., Stewart \& Purcell, 1974).

The difference between the shapes of masking functions have been suggested to indicate two factors involved in masking. Monotonic-shaped masking functions are often interpreted as evidence of integration effects, where the target and the mask effectively become a single stimulus, with the properties of the target hidden by its joint appearance with the mask. U-shaped masking functions are often interpreted as evidence of interruption effects, where the processing of the target stimulus is halted by 
the appearance of the mask. In an extension of these kinds of interpretations, Turvey (1973) and Michaels and Turvey (1979) used the differences in the shape of masking functions to try to distinguish between peripheral and central mechanisms in visual information processing.

Another theory of masking that also draws on the idea that different mechanisms are involved in monotonicand U-shaped masking functions was proposed by Breitmeyer and Ganz (1976) and elaborated by Breitmeyer (1984). They linked U-shaped masking functions to transient inhibitory signals generated by the mask stimulus. The theory suggests that monotonic-shaped masking functions appear when sustained signals generated by the mask are also present and interfere with detection of target properties.

Thus, differences in masking function shapes have played an important role in the interpretation and use of masking. Although many researchers believe that the different-shaped masking functions indicate different mechanisms, we will show below that several quantitative models of masking account for both types of masking functions with a single mechanism. As a result, it is unclear whether in the years of research on backward masking, one mechanism or multiple mechanisms were being investigated. In the following section, we will analyze quantitative models of masking and will show that they can produce both U-shaped and monotonic-shaped masking functions. We show that in these models, the shape of the masking function is related to the strength of masking, so that monotonic-shaped masking functions are produced for strong masks and U-shaped masking functions are produced for weaker masks. We then will report on an experiment that shows that the hypothesized relationship between strength of masking and the shape of the masking function does not always hold.

\section{Model Analysis}

We investigated three quantitatively defined models that account for the U-shaped backward masking function. These include models by Weisstein $(1968,1972)$, Bridgeman (1971, 1978), and Anbar and Anbar (1982). Each of these models has previously been used to account for many other aspects of masking (see, e.g., Francis \& Hermens, 2002). We did not include the model of Francis (1997), because it accounts for $\mathrm{U}$-shaped masking functions only under metacontrast conditions (when the target and the mask spatial contours do not overlap). In the experiment below, we will consider effects from other types of masks that cannot currently be addressed by Francis's (1997) model. Simulation results of the models are shown in Figures 1 and 2. The simulations are based on the original descriptions of the models (Anbar \& Anbar, 1982; Bridgeman, 1978; Weisstein, 1972). Details of the models and parameters can also be found in Francis (2000).

Figure 1A shows masking functions generated by the model of Anbar and Anbar (1982). This model is fairly limited in how it represents the mask stimulus. In particular, the mask is represented only as a single variable that corresponds to the strength of the mask-produced inhibition. Figure $1 \mathrm{~A}$ plots masking functions for curves that vary the intensity of the mask signal, with the target stimulus held fixed. Both U-shaped and monotonic-shaped masking functions can be generated by the model. An analysis of the simulation data shows that U-shaped masking functions appear when the ratio of the mask and the target intensities is 1.09 or less. When the mask-totarget intensity ratio is 1.15 or greater, the masking function is monotonic. The U-shaped and monotonic-shaped masking functions converge for long SOAs. Before the curves converge, the monotonic-shaped masking functions show stronger masking than do any U-shaped masking functions at any fixed SOA. Thus, Anbar and Anbar's model predicts a tight relationship between the shape of the masking function and the overall strength of the mask. Monotonic-shaped masking functions are produced by masks that have strong masking effects, and U-shaped masking functions are produced by masks that have weaker masking effects.

Figure 1B shows masking functions generated by the model of Weisstein (1972). This model can represent both the duration and the intensity of the mask. Each curve in Figure 1B is a masking function generated for a different combination of mask duration and mask intensity. The target intensity (32 units) and duration $(20 \mathrm{msec})$ were fixed for all the simulations. The mask intensity was 0.5 , $1.0,2.0$, or 4.0 times the target intensity and the mask duration was $0.5-4.0$ times the target duration in steps of 0.5 . All combinations of mask intensity and duration were simulated, which made for a total of 32 simulations.

An analysis of the simulation data revealed that monotonic-shaped masking functions were produced for nine of the simulations. (Many of the plotted lines overlap each other in Figure 1B. In addition, two U-shaped masking functions were not plotted in Figure 1B so that the monotonic-shaped functions could be more clearly seen. The properties of these U-shaped functions did not deviate from the general conclusions drawn below.) Monotonicshaped masking functions were produced only for simulations in which the mask intensity ratio was 1.0 or higher. The mask duration ratio was also usually greater than 2.5. In general, monotonic masking functions appeared for longer mask durations and stronger mask intensities; other masks produced U-shaped masking functions.

At each SOA, any monotonic-shaped masking function is, almost always, below any U-shaped masking function. This is consistent with the observation that monotonicshaped masking functions occur for masks with longer durations or larger intensities. There are a few exceptions where the U-shaped and monotonic-shaped curves start to converge at around SOA $=100$. Here, one can see that the furthest-left monotonic-shaped masking function does intersect and rise above a few U-shaped masking functions. However, the crossover is small, and the curves do not deviate much as they converge at longer SOAs.

Thus, both Anbar and Anbar's (1982) model and Weisstein's (1972) model make a similar prediction regarding 

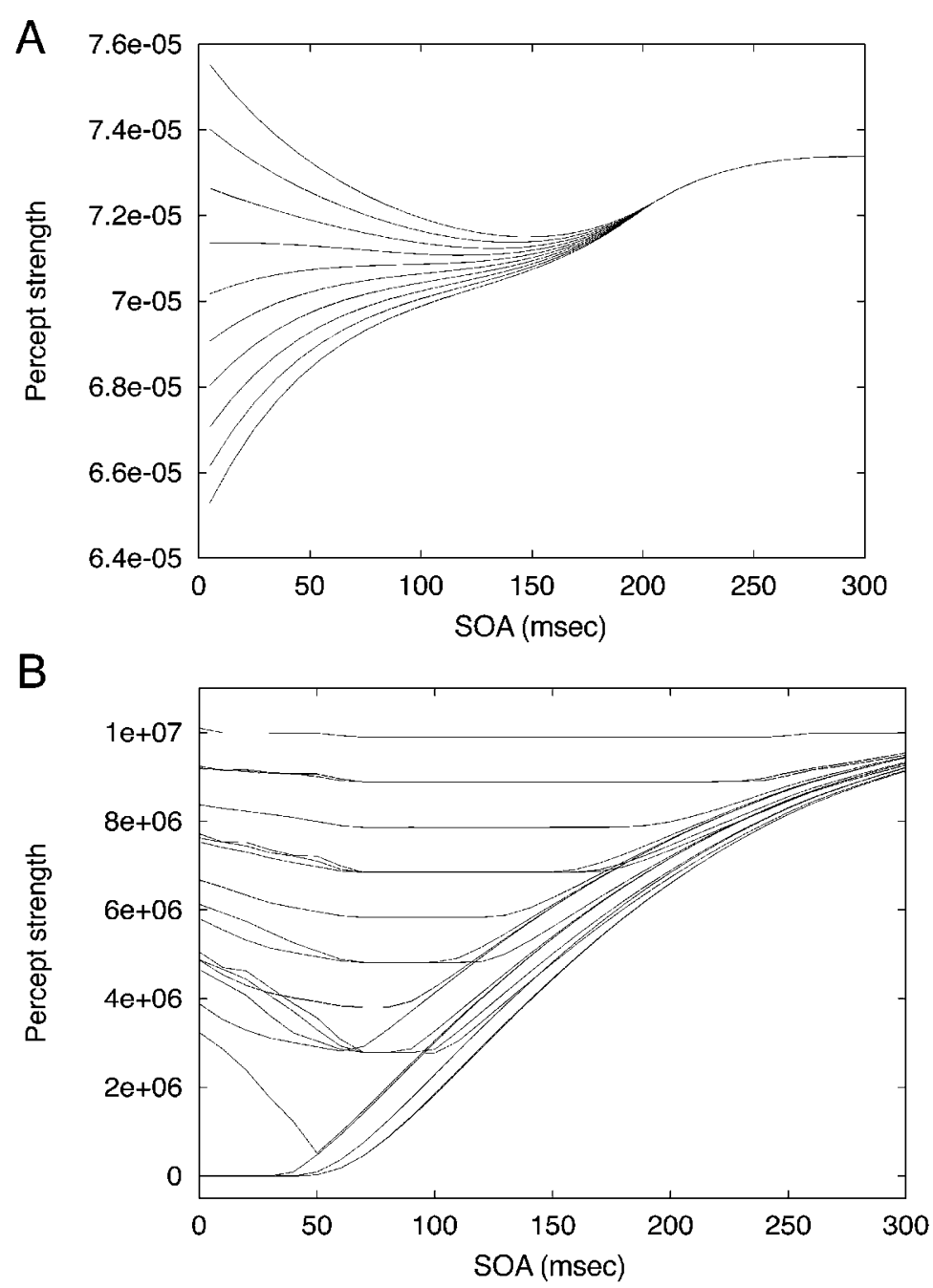

Figure 1. Simulation results for Anbar and Anbar's (1982) and Weisstein's (1972) models. The different curves are for masks with different intensities and/or durations. For almost every stimulus onset asynchrony (SOA), the $\mathrm{U}$-shaped masking functions are above the monotonic-shaped masking functions. (A) Anbar and Anbar's model. (B) Weisstein's (1972) model.

the shape of the masking function and the strength of masking. Both models predict that monotonic-shaped masking functions are produced by masks that generate strong masking effects and that U-shaped masking functions are produced by masks that generate weaker masking effects. Moreover, they predict that for each SOA, any monotonic-shaped masking function should have stronger masking than any U-shaped masking function (although Weisstein's model does allow for very slight deviations from this prediction).

Figure 2 shows masking functions generated by the model of Bridgeman (1978). Figure 2A shows masking functions similar to those generated in Figure 1, with different curves corresponding to different mask durations and intensities. The mask was a pair of inputs on opposite sides of the target stimulus, which corresponds to a metacontrast masking situation. The jitter in the curves corresponds to the use of statistical noise that is introduced into the model computations and oscillatory effects that the model generates. The jitter is small relative to the overall shape of the masking function.

The ratio of the mask to target intensity varied from 0.1 to 2.43 in six multiples of three. The ratio of mask to target duration varied from 0.5 to 2.5 in steps of 0.5 . Thus, there were a total of 30 simulations. Thirteen of the masking functions were monotonic-shaped, whereas the other 17 were U-shaped. As with the simulations of the other models, the monotonic-shaped masking functions were produced by masks of high intensity and/or long duration. For example, all of the masks with intensity ratios of 0.3 or less produced U-shaped masking functions. The pattern of results in Figure 2A is similar 

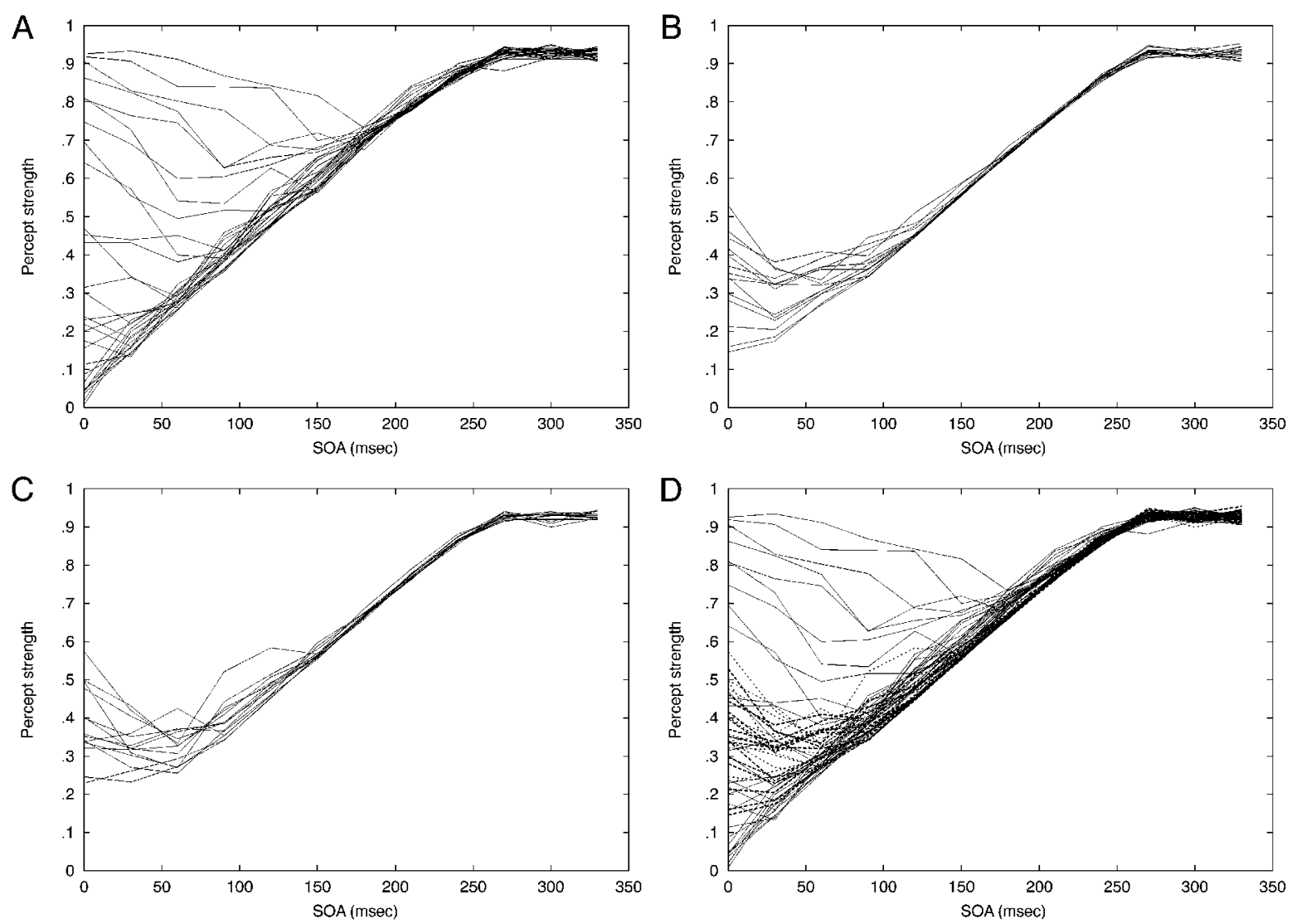

Figure 2. Simulation results for Bridgeman's (1978) model. At each stimulus onset asynchrony (SOA), the U-shaped masking functions are generally above the monotonic-shaped masking functions. (A) The different curves are for metacontrast masks with different mask intensities and/or durations. (B) Different curves correspond to masks with different spacings between foreground elements. (C) Different curves correspond to masks with different random distributions of intensities. (D) This plot combines the data from panels A-C.

to that produced by Anbar and Anbar's (1982) and Weisstein's (1972) models, with monotonic-shaped masking functions having stronger masking than U-shaped masking functions for any given SOA.

Unlike Anbar and Anbar's (1982) model and Weisstein's (1972) model, Bridgeman's (1978) model has a one-dimensional representation of visual space. This representation capability allows the model to distinguish between masks of different spatial properties. To explore this property, we generated masks of various shapes and compared the resulting masking functions.

The first set of simulations used masks made of 1-pixel-wide elements that formed a grating. The spacing between elements was varied from 1 to 14 pixels. Following Bridgeman (1978), the simulation consisted of 30 cells arranged in a circle, so the number of elements in the mask decreased as the spacing between the bars increased. The masking functions produced by these masks are shown in Figure 2B. Although variation in mask spacing did not produce as much variability in masking functions as variation in intensity and duration, the same trends exist. The monotonic-shaped masking functions show stronger masking at every SOA than do the U-shaped masking functions.

Further exploration of shape effects were investigated by generating 20 random mask patterns and their corresponding masking functions. The masking functions are shown in Figure 2C. The results are similar to those for variations in intensity, duration, and spacing. Both U-shaped and monotonic-shaped masking functions were produced. The two curves that produce monotonic-shaped masking functions correspond to stronger masking than do the curves that produce U-shaped masking functions. This strength relationship holds for essentially every SOA.

Finally, Figure 2D combines the masking functions across all the mask types. The solid lines correspond to the masking functions in Figure 2A, where the mask was a metacontrast mask of varying intensity and duration. The heavier dashed line corresponds to the masking functions in Figure 2B, where the mask was a grating with various spaces between the bar elements. The dotted line corresponds to the masking functions in Figure $2 \mathrm{C}$, where the masks were different random patterns. Although there are a few exceptions, the general trend in Figure 2D is for the 
monotonic-shaped masking functions to correspond to stronger masking at every SOA, as compared with any U-shaped masking function. When an exception does occur, it is at short SOAs, where many curves are crowded together. Some of the crossovers were probably due to the internal jitter produced by noise in the model.

A consideration of all of the data in Figure 2 indicates that in Bridgeman's (1978) model, monotonic-shaped masking functions appear for masks that generate strong masking effects and U-shaped masking functions appear for masks that generate weaker masking effects. For any given SOA, a monotonic-shaped masking function has stronger masking than does a U-shaped masking function. This relationship holds regardless of what mask properties (intensity, duration, or shape) are varied between the two masking functions.

The relationship between masking function shape and masking strength is, thus, a prediction of all of the models. It should be noted that there are currently no experimental data that go against this prediction. As Kolers (1962) and many subsequent studies have noted, monotonicshaped masking functions are usually seen with masks that generate strong masking effects, and U-shaped masking functions are usually seen with masks that generate weaker masking effects. For those studies that systematically varied mask intensity or duration (e.g., Breitmeyer, 1978; Spencer \& Shuntich, 1970; Weisstein, 1972), the U-shaped curves always showed weaker masking than did monotonic-shaped curves. A possibility suggested by the models is that the relative strength of masking generated by a given mask entirely determines the shape of the masking function.

Significantly, the model of Bridgeman (1978) suggests that the relationship between mask strength and masking function shape holds regardless of the type of mask, as Figure 2D indicates. The models of Anbar and Anbar (1982) and Weisstein (1972) do not distinguish between masks with different spatial properties. This has long been recognized as a limitation of these models (e.g., Weisstein, 1972). Perhaps, for Anbar and Anbar's model, one could propose that different-shaped masks simply give rise to different mask signal strengths. Likewise, for Weisstein's (1972) model, one could propose that differentshaped masks give rise to different mask signal strengths and durations. If these minimal modifications to the models were true, this would indicate that backward masking may be explained by relatively few model properties.

After an extensive literature search, we have failed to find any experimental studies that fixed the target and task properties while comparing masking functions produced by different types of masks. For example, although a pattern mask may produce a monotonic-shaped masking function and a metacontrast mask may produce a U-shaped masking function, no one has ever checked to see whether the pattern mask generally produced stronger masking effects than did the metacontrast mask. If monotonic-shaped masking functions always appear only with stronger masking effects and U-shaped mask- ing functions always appear only with weaker masking effects, this would be strong support for the properties of these models. On the other hand, if the shape of the masking function is independent of the overall strength of masking effects, this would indicate that all of the models have missed an important component of the systems that are involved in masking.

To recapitulate, the models predict that any $\mathrm{U}$-shaped masking function must show weaker masking than does any monotonic-shaped masking function, regardless of what types of masks are used in the different conditions. The one restriction is that the variation in mask types must be the only difference across the conditions. The target and the task must be unchanged for the comparison to be valid. The existing literature on masking, although vast, has not explored variation in mask type with a fixed target and task.

\section{PSYCHOPHYSICAL EXPERIMENT}

We investigated backward masking of a fixed target and task with two types of masks. One type was a metacontrastlike mask with four small lines around, but not overlapping, the target (see also Enns \& Di Lollo, 2000). Metacontrast masks are known to produce U-shaped masking functions (Breitmeyer, 1984), provided the intensity and duration of the mask are not dramatically larger than those of the target. The other mask type is called a shinethrough mask, which consisted of a grating made of 25 aligned verniers. Herzog, Koch, and Fahle (2001) noted that for short SOAs, this type of mask can obliterate the visibility of the target. On the basis of the experimental work in earlier studies, we expected the particular shinethrough mask we used to produce a monotonic-shaped masking function.

\section{Method}

Stimuli. The stimuli were displayed on an analog monitor (HP 1332 A) controlled by a Power Macintosh computer via fast 16-bit D/A converters (1-MHz pixel rate). Refresh time of the monitor was $5 \mathrm{msec}$, and at stimulus offset the phosphor of the monitor decayed to $4 \%$ of its maximum intensity in less than a millisecond. The subjects observed the stimuli from a distance of $2 \mathrm{~m}$ in a room illuminated dimly by a background light $(0.5 \mathrm{~lx})$. Luminance of the stimuli was around $80 \mathrm{~cd} / \mathrm{m}^{2}$. Before stimulus presentation proper, a fixation dot in the middle of the screen and four markers at the corners of the monitor appeared.

In all the experiments, a vernier served as a target element and was displayed for $10 \mathrm{msec}$ in the middle of the screen. The top and bottom bars of the vernier were shifted symmetrically around the middle point in a direction that changed randomly across trials. Each of the two vernier segments was 600 arcsec long and about $30 \operatorname{arcsec}$ wide. The top and bottom segments were separated by a small vertical gap of 60 arcsec. The vernier was followed by either the metacontrast or the shine-through mask, which lasted for $300 \mathrm{msec}$ (see Figure 3).

The metacontrast mask consisted of four flanking bars, which appeared at the upper, lower, left, and right ends of the vernier. Each flanking bar had a length of 400 arcsec and a width of about 30 arcsec. The position of the four masking elements was determined individually, with the aim of satisfying two requirements. First, performance for an SOA of $10 \mathrm{msec}$ should be affected only weakly by 
A

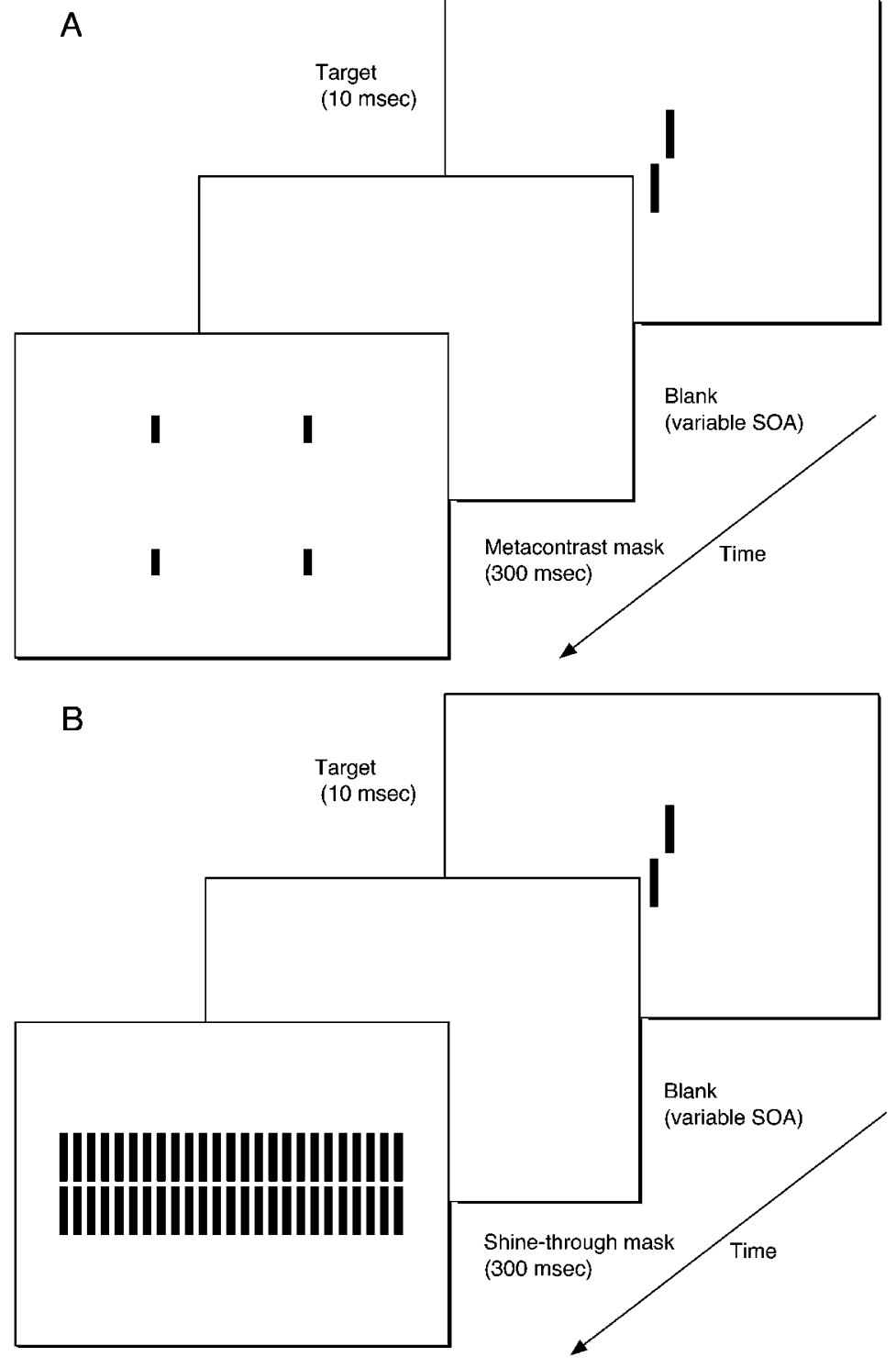

Figure 3. Schematizations of trials in the psychophysical experiment. The target stimulus was a vernier target that was presented for $10 \mathrm{msec}$. The mask followed the target by a variable stimulus onset asynchrony (SOA) and was presented for 300 msec. (A) The metacontrast mask consisted of four bars that spatially flanked the vernier target but did not overlap it. (B) The shine-through mask consisted of a grating consisting of 25 aligned verniers.

the metacontrast mask. Second, for SOAs in the range of 40-70 msec, strong masking should occur. Mask elements were separated from the center by a distance in the range of 200-600 arcsec in the horizontal direction and about 630 arcsec in the vertical direction, about the length of a vernier segment. Once the appropriate element separation was found for an observer, it was fixed for the entirety of the experiment.

The shine-through mask consisted of a grating with 25 elements. Except for offset, the spatial parameters of the mask grating elements and the target vernier were the same. The horizontal spacing between grating elements was 200 arcsec. The middle element of the grating and the vernier both appeared in the center of the screen.

Procedure. The observer's task was to report whether the bottom segment of the vernier was shifted to the left or the right, relative to the top segment. After each trial, the observer received auditory feedback to indicate whether the response was correct or incorrect. Masking was measured by finding a $75 \%$ correct threshold spatial offset of the vernier via an adaptive staircase procedure (PEST; Taylor \& Creelman, 1967).

Eight or nine SOAs were run for each observer for each mask type. The adaptive procedure was used to find the threshold for one SOA and mask type at a time. The order of SOAs was randomized within each observer, to reduce possible order effects. For 3 observers, the threshold was measured twice for each SOA. After every condition had been tested once, the order of conditions was reversed for the second measurements, in order to at least partly compensate for possible learning effects. The threshold was mea- 
sured only one time for Observer G.F. The metacontrast and shinethrough masks were presented in alternating threshold measures.

All the observers had normal or corrected-to-normal acuity, as determined by the Freibuger acuity test (Bach, 1995). The first author and 3 naive observers (from the University of Bremen) participated.

\section{Results}

Figure 4 plots masking functions for the metacontrast and shine-through masks. A separate graph is shown for each observer. Each graph plots the threshold of the vernier offset against SOA. Larger thresholds indicate stronger masking. For observer M.H., the vernier was invisible for the shine-through mask with an SOA of $10 \mathrm{msec}$, and no threshold could be found. Since this indicates very strong masking, it did not seem prudent to discard this case from the results. As a compromise, a large threshold value of 150 was assigned to this condition. This is just a bit larger than the actual threshold values for observers that could make vernier discriminations in this condition. None of our conclusions depend on this compromise.

As was expected, for each observer, the metacontrast mask produced an inverted U-shaped masking function. The plot is an inverted-U instead of a U-shape because the $y$-axis plots the threshold separation of the vernier and larger thresholds indicate stronger masking effects.

The shine-through mask produced monotonic-shaped masking functions, except for small fluctuations that were likely due to sampling variation. The strongest masking occurred for the smallest SOA, and as the mask followed the target by longer delays, the vernier discrimination task became easier. The one exception was for Observer G.F., whose masking function was not technically monotonic. For every observer, the U-shaped and monotonic-shaped masking functions intersected. Figure 5 combines the data in Figure 4 across all the observers. The error bars indicate variability in the thresholds across observers. For the combined data, the U-shaped curve and the monotonic curve intersected at an SOA of approximately $30 \mathrm{msec}$.

\section{Discussion}

The experimental data contradict the prediction of the models. The models predict that for a fixed target, a fixed task, and any SOA, a mask that produces a U-shaped masking function should produce weaker masking effects than does a mask that produces a monotonic-shaped mask-
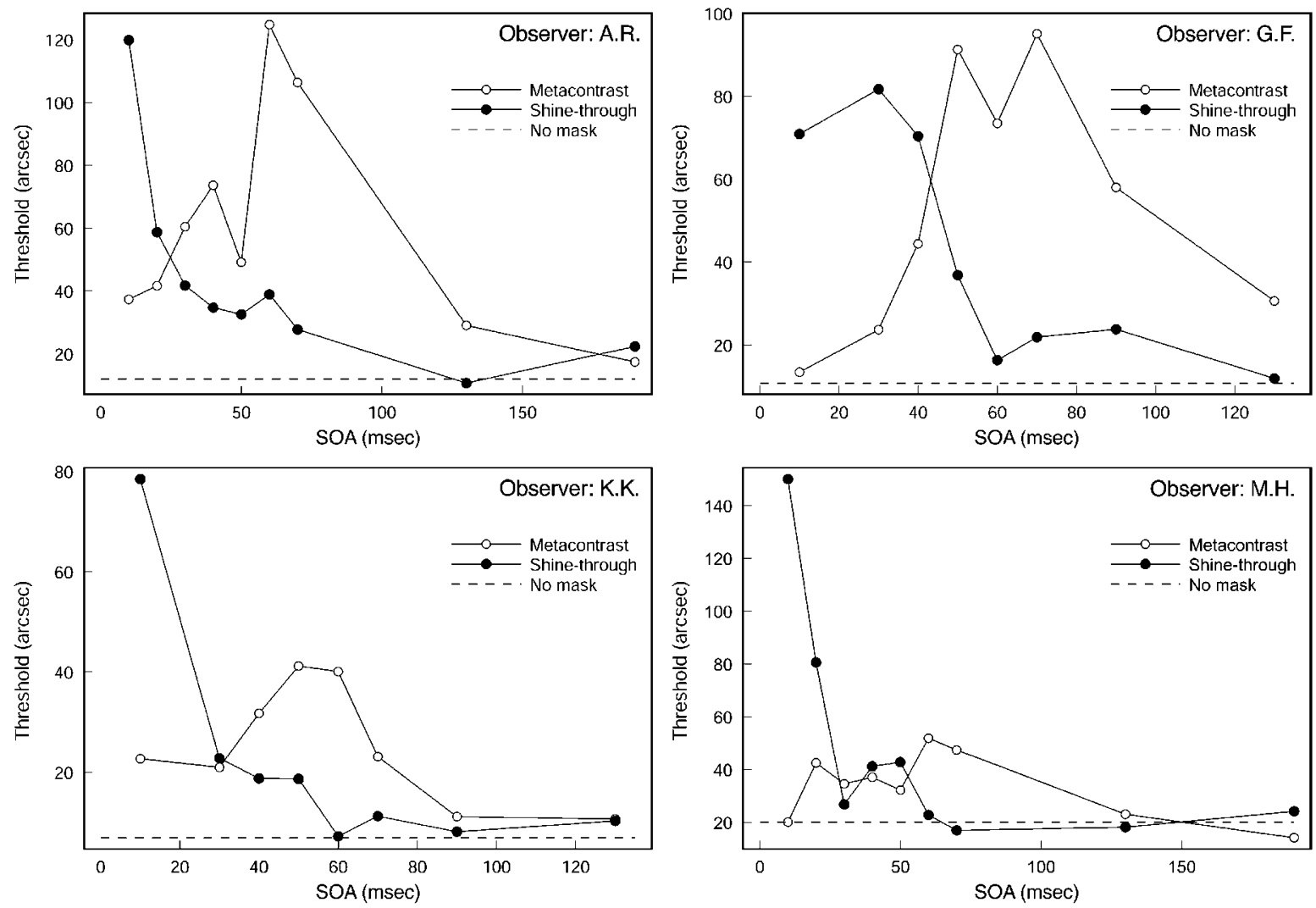

Figure 4. Masking functions for a metacontrast and a shine-through mask for 4 different observers. The dashed horizontal line indicates the threshold for an unmasked vernier. The thresholds for the shine-through mask generally decrease as stimulus onset asynchrony (SOA) increases, which indicates that the strongest masking occurs for small SOAs. For the metacontrast mask, the expected inverted $U$-shaped threshold curve occurs, which indicates that masking is strongest for intermediate SOAs. The two masking curves intersect, which is in violation of the models. 


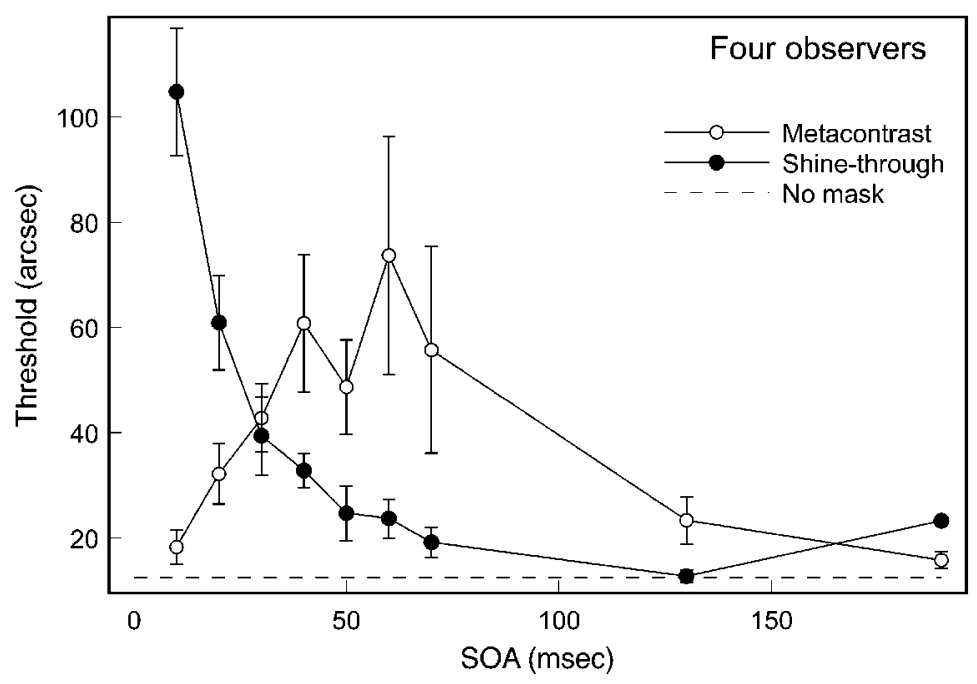

Figure 5. Averaged data across all the observers. The error bars show the standard errors across observers. SOA, stimulus onset asynchrony.

ing function. The data do not support this prediction. The data for each observer show that at short SOAs, the experimentally found monotonic-shaped masking function has stronger masking than does the U-shaped masking function. At SOAs between 30 and $100 \mathrm{msec}$, the experimentally found $U$-shaped masking function exhibits stronger masking than does the monotonic-shaped masking function. This indicates that contrary to the properties of the models, the shape of the masking function is not exclusively related to the overall strength of masking.

\section{CONCLUSIONS}

Quantitative models of backward masking predict that U-shaped masking functions will have weaker masking than do monotonic-shaped masking functions. Lots of experimental data in a variety of contexts have shown this prediction to be valid (e.g., Kolers, 1962). However, the experimental data in this article demonstrate that there is at least one situation in which this prediction is not correct.

Our data also offer problems for the transient-sustained model proposed by Breitmeyer and Ganz (1976) and elaborated by Breitmeyer (1984). This model proposes two ways that the mask interacts with the target. In this model, U-shaped masking functions appear due to an interaction of transient inhibition from the mask with sustained responses generated by the target. Monotonic-shapedmasking functions appear when sustained inhibition from the mask also interacts with sustained responses generated by the target. Thus, in their theory, monotonic-shaped masking functions will always be associated with stronger masking than are U-shaped masking functions, because the monotonic shape will appear when both transient and sustained inhibitory effects are present. The experiment reported in this article shows that this prediction is not valid in at least one situation.
One way to promote model development, either to extend the current models or to create new models, is to look for additional situations in which U-shaped masking functions show stronger masking than do monotonic-shaped masking functions. If such cases are rare and seem to appear only for a few particular combinations of masks, the models may only need to be elaborated to explain the nature of the particular effects of those masks. On the other hand, if U-shaped masking functions are often found to show stronger masking than do monotonic-shaped masking functions, the models are more seriously flawed. Then, either the models will need substantial elaboration, or they should be rejected outright.

\section{REFERENCES}

ANBAR, S., \& ANBAR, D. (1982). Visual masking: A unified approach. Perception, 11, 427-439.

BACH, M. (1995). Der Freiburger Visustest: Automatisierte Sehschärfebestimmung. Der Ophthalmologe, 92, 174-178.

BRAFF, D. L., \& SACCUZzo, D. P. (1981). Information processing dysfunction in paranoid schizophrenia: A two-factor deficit. American Journal of Psychiatry, 138, 1051-1056.

BREITME YER, B. G. (1978). Metacontrast masking as a function of mask energy. Bulletin of the Psychonomic Society, 12, 50-52.

BreitMeyer, B. G. (1984). Visual masking: An integrative approach. New York: Oxford University Press.

BreitMeyer, B. G., \& GANZ, L. (1976). Implications of sustained and transient channels for theories of visual pattern masking, saccadic suppression, and information processing. Psychological Review, 83, $1-36$.

Breitmeyer, B. G., \& Ogmen, H. (2000). Recent models and findings in visual backward masking: A comparison, review, and update. Perception \& Psychophysics, 62, 1572-1595.

BRIDGEMAN, B. (1971). Metacontrast and lateral inhibition. Psychological Review, 78, 528-539.

BRIDGEMAN, B. (1978). Distributed sensory coding applied to simulations of iconic storage and metacontrast. Bulletin of Mathematical Biology, 40, 605-623.

ENNS, J. T., \& Di Lollo, V. (2000). What's new in visual masking? Trends in Cognitive Sciences, 4, 345-352. 
FRANCIS, G. (1997). Cortical dynamics of lateral inhibition: Metacontrast masking. Psychological Review, 104, 572-594.

FrancIS, G. (2000). Quantitative theories of metacontrast masking. Psychological Review, 107, 768-785.

Francis, G., \& Hermens, F. (2002). Comment on "Competition for consciousness among visual events: The psychophysics of reentrant visual processes" (Di Lollo, Enns, \& Rensink, 2000). Journal of Experimental Psychology: General, 131, 590-593.

Green, M. F., Nuechterlein, K. H., \& Mintz, J. (1994). Backward masking in schizophrenia and mania: I. Specifying a mechanism. Archives of General Psychiatry, 51, 939-944.

Hellige, J. B., Walsh, D. A., LaWrence, V. W., \& Prasse, M. (1979). Figural relationship effects and mechanisms of visual masking. Journal of Experimental Psychology: Human Perception \& Performance, 5, 88-100.

HerzoG, M. H., Koch, C., \& FAHLE, M. (2001). Shine-through:Temporal aspects. Vision Research, 41, 2337-2346.

KolERS, P. A. (1962). Intensity and contour effects in visual masking. Vision Research, 2, 277-294.

Michaels, C. F., \& Turvey, M. T. (1979). Central sources of visual masking: Indexing structures supporting seeing at a single, brief glance. Psychological Research, 41, 1-61.

SCHILlER, P. H. (1965). Metacontrast interference as determined by a method of comparison. Perceptual \& Motor Skills, 20, 279-285.
SLAGHUis, W. L., \& BAKKER, V. J. (1995). Forward and backward visual masking of contour by light in positive- and negative-symptom schizophrenia. Journal of Abnormal Psychology, 104, 41-54.

SPENCER, T. J., \& SHUNTICH, R. (1970). Evidence for an interruption theory of backward masking. Journal of Experimental Psychology, 85, 198-203.

Stewart, A. L., \& Purcell, D. G. (1974). Visual backward masking by a flash of light: A study of U-shaped detection functions. Journal of Experimental Psychology, 103, 553-566.

TAYlor, M. M., \& CREelman, C. D. (1967). PEST: Efficient estimates on probability distributions. Journal of the Acoustical Society of America, 41, 782-787.

TURVEY, M. T. (1973). On peripheral and central processes in vision: Inferences from an information processing analysis of masking with patterned stimuli. Psychological Review, 80, 1-52.

Weisste IN, N. (1968). A Rashevsky-Landahl neural net: Simulation of metacontrast. Psychological Review, 75, 494-521.

Weisstein, N. (1972). Metacontrast. In D. Jameson \& L. M. Hurvich (Eds.), Handbook of sensory physiology: Vol. 7. No. 4. Visual psychophysics (pp. 233-272). Berlin: Springer-Verlag.

(Manuscript received August 12, 2002; revision accepted for publication December 20, 2002.) 